\title{
Catcher Pole Scleral Fixation for Repositioning of Dislocated IOL in Vitreous Chamber
}

\section{A Scupola ${ }^{1,3}$, Maria Ludovica Ruggeri ${ }^{1 *}$, A Savastano $^{2,3}$, MA Blasi $^{1,3}$ and $S \operatorname{Rizzo}^{2,3}$}

${ }^{1}$ UOC Oncologia Oculare, Fondazione Policlinico Universitario A. Gemelli IRCCS,

Rome, Italy

${ }^{2}$ UOC Oculistica, Fondazione Policlinico Universitario A. Gemelli IRCCS, Rome, Italy

${ }^{3}$ Sezione di Oculistica, Università Cattolica del Sacro Cuore, Rome, Italy

*Corresponding Author: Maria Ludovica Ruggeri, UOC Oncologia Oculare,

Fondazione Policlinico Universitario A. Gemelli IRCCS, Rome, Italy.
Received: February 26, 2021

Published: May 31, 2021

(C) All rights are reserved by Maria Ludovica

Ruggeri., et al.

\section{Abstract}

Purpose: To describe a new surgical instrument for IOL repositioning in scleral fixation procedures designed to help the surgeon to catch the haptics of the dislocated IOL and then handle them to scleral fixation.

Methodology: Twenty-one eyes of 21 patients who underwent IOL repositioning procedure through the handling of this new surgical tool were enrolled in this observational study. The instrument is a flute needle with several side slits that allow the passage of a looped suture (10-0 polypropylene ethicon Prolene EH7896) in order to obtain a tool similar to a catcher pole to engage the haptics and ease the handling during scleral fixation procedure.

Results: This procedure has shown to be effective in terms of optic rehabilitation and surgical outcomes.

Conclusion: The use of this tool simplifies IOL repositioning during sutured scleral fixation technique representing a valuable help for the surgeon in obtaining satisfying anatomical and visual outcomes.

Keywords: Intraocular Lens Dislocation; Intraocular Lens Repositioning; Personalized Medicine; Innovative Biotechnologies; Scleral Fixation; Vitrectomy

\section{Introduction}

Intraocular lens (IOL) dislocation into vitreous cavity is an infrequent but serious complication of cataract surgery that may occur at the time of surgery to years. Despite improvements of surgical techniques, the management of dislocated IOLs remains a challenge and every surgical case needs a careful evaluation in order to establish the best course of treatment. Recognizing the common surgical purpose, different approaches may be practicable to achieve optical rehabilitation including removal, replacement or repositioning of the dislocated intraocular lens [1]. Although ex- planation/reimplantation still remains the best surgical option in some cases, the repositioning of the intraocular lens is considered as an attractive and suitable procedure allowing, in most cases, a lower loss of endothelial cells, less discomfort for the patient and a more rapid visual recovery [2]. During the years, many surgical procedures of dislocated IOL repositioning have been proposed, including techniques of iris suture fixation, scleral suture fixation and more recently, techniques of intrascleral haptic fixation [3]. Each procedure has its own advantages and disadvantages and the choice of surgical technique depends on the clinical features 
as well as surgeon's preferences [4]. In this article, we describe a technique of IOL repositioning using a new instrument designed to help the surgeon to catch the haptics of the dislocated IOL and then handle them to scleral fixation.

\section{Case Report}

This study adhered to the tenets of the Declaration of Helsinki. All enrolled patients provided informed consent. Twenty-one eyes of 21 patients with vitreous dislocation of the intraocular lens were enrolled in this observational study. All the surgeries were performed under peribulbar anesthesia by the same surgeon (A.S.) according to the following steps: after localized peritomy of the conjunctiva, two partial-thickness limbus-based triangular scleral flaps $(2.5 \times 2.5 \mathrm{~mm})$ in the opposite side at 3 and 9-o'clock positions were prepared with the aim to minimize the risk of postoperative suture exposure. Bimanual 3-port pars plana 25-gauge vitrectomy (Constellation Vitrectomy System -Alcon Laboratories, USA) was performed in every case, with a careful shaving of the vitreous base and removal of the anterior vitreous. A 25 Chandelier Endoillumination for Panoramic Viewing (Alcon Laboratories- USA) was used During Vitreous Surgery. In case of IOL luxated within the lens capsule, the haptics end of the IOL were cleared from the capsular bag using the vitrectomy probe. If present, capsular tension ring was separated from the IOL and removed through the anterior chamber. Next steps were aimed at hooking the dislocated IOL. To simplify the engagement of the haptics and then to guide them to a specific point for scleral fixation, a new tool was designed. The instrument is a flute needle 25 gauge with several side slits necessary for passage of a long curved looped needle (10-0 polypropylene ethicon Prolene EH7896) in order to obtain a tool similar to a catcher pole (Figure 1). The instrument was introduced into the vitreous cavity through a sclerotomy incision into the scleral flap at a distance of $2 \mathrm{~mm}$ from the limbus. Considering the possible slippage of the suture during the instrument introduction through the sclera, the size of the loop was initially realized bigger than required and then adjusted and downsized inside the vitreous cavity (Figure 2). Once introduced the "catcher pole" in the vitreous chamber, the IOL was mobilized with a vitreoretinal 25-gauge forceps and with bimanual technique the haptic was captured by snaring within the loop. To avoid slippage of the suture, the end of the haptic was grasped with the vitreous forceps while pulling the suture. The suture was temporarily tied until the opposite haptic was engaged and sutured to the sclera before positioning the IOL at the center with the same fixation technique. Both sutures were strictly tied onto the ciliary sulcus. To increase the IOL stability and reduce the risk of postoperative haptic slippage with subsequent lens subluxation, in the last cases we revised our technique passing a deep second suture within the scleral flap in order to strengthen the haptic connection to the sclera (Supplemental Video file).

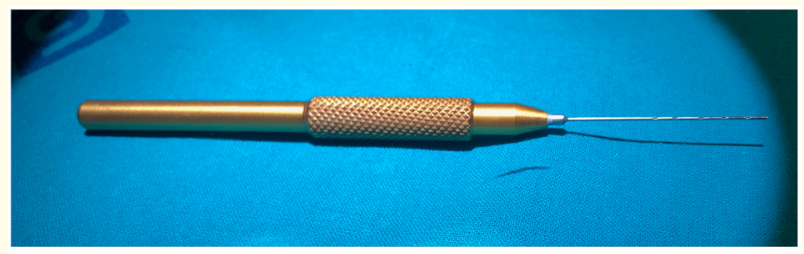

Figure 1: The "Catcher pole" surgical instrument. The tool is a flute needle 25 gauge with several side slits necessary for passage of curved looped needle in order to obtain a tool similar to a catcher pole.

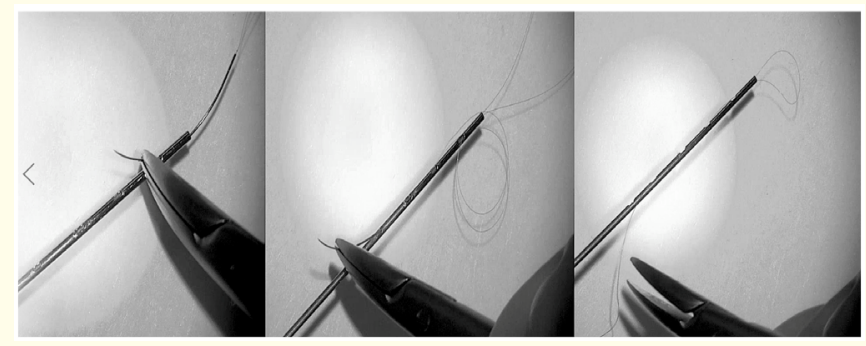

Figure 2: Surgical preparation of the catcher pole. The curved needle is passed through the side slits and suture is adjusted in order to obtain a loop to capture the dislocated IOL in the vitreous chamber.

\section{Discussion}

Intraocular lens (IOL) dislocation is an uncommon complication of cataract surgery. In early cases, the dislocation is often "out of bag" and related to an unsuitable IOL placement, often in surgery complicated by posterior capsule rupture and/or zonular dehiscence. Unlike early lens dislocation, late dislocation usually occurs several years after uneventful cataract surgery and is mostly observed as "in the bag" dislocation where the IOL is still contained 


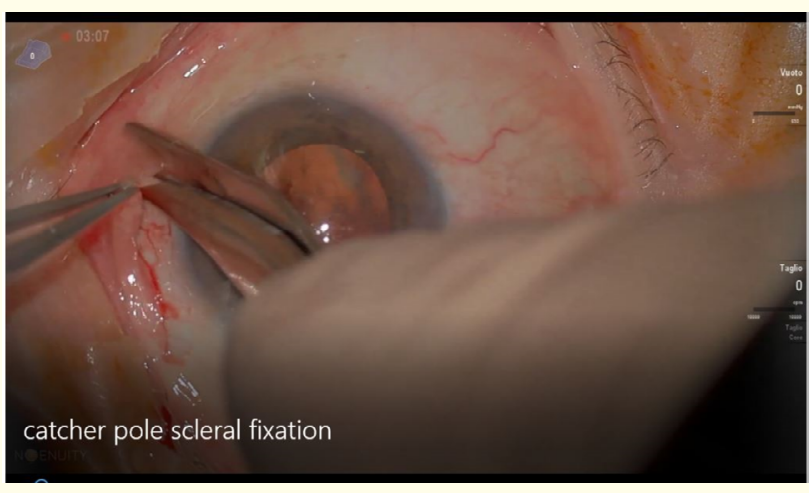

inside the capsular bag. In-bag IOL dislocation seems the result of a progressive weakness of the fibers of the Zinn zonule and is often associated with Pseudoexfoliation (PEX) syndrome, or other predisposing disorders, as trauma, previous vitreoretinal surgery, retinitis pigmentosa, pathologic myopia, Marfan syndrome [5]. Since IOL explanation/reimplantation in most cases may be more traumatic than repositioning the IOL, theoretically this option represents a best surgical approach to obtain visual recovery and structural stability. Scleral-fixated IOLs (SFIOLs), first described by Malbran in 1986, have been widely used for surgical correction of aphakia and for suturing a luxated IOL without lens extraction. During the past years different techniques for repositioning posteriorly dislocated intraocular lenses have been described according to the material used, method of suturing and ways of placing suture knots [6-9]. Regardless of the technique, SFIOL using non-absorbable sutures are commonly accepted skills of IOL replacement in cases of inadequate capsular support, resulting in good outcomes and a low risk of complications in the early postoperative period [10]. The main disadvantage of SFIOLs suture techniques is possible recurrent IOL dislocation due to suture erosion and breakage, generally occurring years after the surgery, due to a slowly biodegradation of the sutures. Suture breakage has been reported in $27.9 \%$ of cases 6 years after IOL suturing using 10-0 polypropylene sutures [11]. To avoid suture related complications, recently Scharioth., et al. described a technique of sutureless scleral fixation of standard 3-Pieces Posterior Chamber Intraocular lens (PCIOL) in ciliary sulcus through incarcerating the haptics in a limbus-parallel scleral tunnel overcoming the risk of suture related complications [12].
Even if intrascleral haptic fixation techniques have recently been increasing in acceptance, the long-term outcome of intrascleral IOL fixation has not yet been rated satisfactorily. The most important concern is the stability of the haptics of the IOL and potential risk of postoperative hypotony [3]. Suture fixation to the sclera remains a valid therapeutic option for IOL repositioning, with similar outcomes in visual acuity and IOL stability compared to sutureless flanged Intraocular Lens Fixation [13]. Moreover, although singlepiece IOLs that have dislocated into the vitreous generally need to be exchanged, it should be considered that suture scleral fixation techniques can be eventually used also for repositioning of foldable single piece IOL, that have currently become the most used IOL in cataract surgery. Recently, Tamburelli., et al. described a technique using a loop retinal scraper (finesse Flex Loop DSP Alcon Grieshaber AG) with a retractable wired loop. Despite conceptually similar to ours, in this technique, catcher pole is used with the purpose of catching and externalizing the tip of the IOL haptic [14]. In our technique "Catcher Pole" is used to facilitate the passage of the loop suture through the haptics. The main advantage of using the tool is to permit an easier loop preparation through the thread passage within the needle side holes. Moreover, due to its elongated shape, "Catcher Pole" can be efficiently used directly in the vitreous chamber, making the haptic engagement maneuver easier. In view of these reasons, "Catcher Pole" scleral fixation is an appropriate surgical option suitable for IOL implantation in eyes without sufficient capsular support. The main weakness of the technique is related to the possible breakage of the suture during follow-up. However, the occurrence of this late complication could be reduced by a two-point scleral suture of each haptic minimizing postoperative complications [15].

\section{Conclusion}

In conclusion the "catcher pole" scleral fixation technique offers good results in terms of optical and anatomical stability representing a good surgical option for IOL repositioning and optical rehabilitation in adults. Although at this moment the tool is not commercially available, it can be easily realized making the suture fixation procedure easier and faster.

\section{Acknowledgements}

The authors would like to thank Dr William E. Smiddy for his invaluable comments and suggestions for the manuscript. 


\section{Conflict of Interest}

The authors have no financial disclosures.

\section{Bibliography}

1. Besek NK., et al. "Comparative evaluation of re-use or replacement of dislocated 3-piece intraocular lenses with a scleral fixation technique". Journal Français D'Ophtalmologie 43.2 (2020): 139-144.

2. Smiddy WE and Flynn Jr HW. "Management of dislocated posterior chamber intraocular lenses". Ophthalmology 98.6 (1991): 889-894.

3. Yamane S., et al. "Flanged Intrascleral Intraocular Lens Fixation with Double-Needle Technique". Ophthalmology 124.8 (2017): 1136-1142.

4. Wagoner MD., et al. "Intraocular lens implantation in the absence of Capsular Support. Ophthalmic technology assessment" (2003): 840-859.

5. Rodrigo BJ., et al. "Intraocular Lens Subluxation in Marfan Syndrome". The Open Ophthalmology Journal is an Open 8 (2014): 48-50.

6. Yang S., et al. "Surgical management of intraocular lens dislocation: A meta-analysis" 14.2 (2019).

7. Caporossi T., et al. "IOL repositioning using iris sutures: a safe and effective technique". International Journal of Ophthalmology 12.12 (2019): 1972-1977.

8. Sella S., et al. "Pars plana vitrectomy for posteriorly dislocated intraocular lenses: risk factors and surgical approach". International Ophthalmology 41.1 (2021): 221-229.

9. Chan CK., et al. "Management of dislocated intraocular implants". Ophthalmology Clinics of North America 14.4 (2001): 681-693.

10. Maguire AM., et al. "Scleral loop fixation for posteriorly dislocated intraocular lenses. Operative technique and long-term results". Archives of Ophthalmology 109.12 (1991): 17541758.

11. Brendan J Vote., et al. "Long-term outcome of combined pars plana vitrectomy and scleral fixated sutured posterior cham- ber intraocular lens implantation". American Journal of Ophthalmology 141.2 (2006): 308-312.

12. Scharioth GB., et al. "Intermediate results of sutureless intrascleral posterior chamber intraocular lens fixation”. Journal of Cataract and Refractive Surgery - LWW Journals 36 (2010): 254-259.

13. Do JR., et al. "A 1-year Prospective Comparative Study of Sutureless Flanged Intraocular Lens Fixation and Conventional Sutured Scleral Fixation in Intraocular Lens Dislocation". Ophthalmologica (2020).

14. Tamburrelli $C$ and Tamburrelli AC. "Sutureless Scleral Fixated IOL: The "Catcher Pole" Technique". Clinical Ophthalmology 15 (2021): 121-128.

15. Stem MS., et al. "Scleral- Fixated Intraocular Lenses: Past and Present”. Journal of VitreoRetinal Diseases 1.2 (2017): 144-152.

\section{Volume 4 Issue 6 June 2021 (C) All rights are reserved by Maria Ludovica Ruggeri., et al.}

